\title{
Posição do sesamoide lateral em relação ao segundo metatarso em pés com e sem hálux valgo*
}

\section{Lateral Sesamoid Position Relative to the Second Metatarsal in Feet with and without Hallux Valgus}

\author{
Daniel Gonçalves Machado ${ }^{1}$ Elaine da Silva Gondim ${ }^{1}$ José Carlos Cohen ${ }^{1}$ \\ Luiz Eduardo Cardoso Amorim ${ }^{1}$ \\ 1 Serviço de Cirurgia do Pé e Tornozelo, Hospital Universitário \\ Clementino Fraga Filho (HUCFF), Universidade Federal do Rio de \\ Janeiro, Rio de Janeiro, RJ, Brasil \\ Address for correspondence José Carlos Cohen, Serviço de Cirurgia \\ do Pé e Tornozelo, Hospital Universitário Clementino Fraga Filho \\ (HUCFF), Universidade Federal do Rio de Janeiro, Rio de Janeiro, RJ, \\ Brasil (e-mail: cohenorto@yahoo.com). \\ Rev Bras Ortop 2019;54:165-170.
}

\begin{abstract}
Resumo
Objetivo Determinar se os sesamoides migram lateralmente nos pés com hálux valgo ou se apenas aparentam deslocar-se, mantendo sua relação com as demais estruturas do antepé.

Métodos Foram avaliadas radiografias na incidência anteroposterior com carga dos pés de 80 pacientes ( 94 pés) entre o período de 2015 e 2016. Dessas, 48 tinham ângulo de hálux valgo maior do que $15 \circ$ (grupo hálux valgo) e 46 tinham ângulo de hálux valgo menor do que 15。 (grupo controle). Foram medidas as distâncias da cabeça do primeiro metatarso e do sesamoide lateral ao eixo do segundo metatarso. Posteriormente, foram medidos os coeficientes dessas distâncias pelo comprimento do segundo metatarso, a fim de se ajustarem aos diferentes tamanhos de pés.

Resultados Tanto a medida absoluta quanto a medida relativa da cabeça do primeiro metatarso ao segundo metatarso foram significativamente diferentes nos dois grupos, tiveram correlação positiva com os ângulos de hálux valgo e intermetatarsal. Contudo, nem a distância absoluta nem a relativa do sesamoide lateral ao segundo metatarso foram diferentes nos dois grupos, bem como não se correlacionaram com os ângulos de hálux valgo e inter-metatarsal.

Palavras-chave

- hallux valgus

- ossos do metatarso

- radiografia

- análise estatística

Conclusão Apesar do desvio medial do primeiro metatarso no hálux valgo, o sesamoide mantém sua relação com o segundo metatarso no plano transverso. Esse aparente deslocamento lateral pode levar a interpretação equivocada dessas radiografias. Tal fato é de suma importância no pré-, peri- e pós-operatório dos pacientes com hálux valgo.
\end{abstract}

Estudo desenvolvido no Serviço de Cirurgia do Pé e Tornozelo, Hospital Universitário Clementino Fraga Filho (HUCFF), Universidade Federal do Rio de Janeiro, Rio de Janeiro, RJ, Brasil. Publicado originalmente por Elsevier Ltda. received

November 1, 2017

accepted

December 14, 2017
DOI https://doi.org/

10.1016/j.rbo.2017.12.018. ISSN 0102-3616.
Copyright $@ 2019$ by Sociedade Brasileira License terms de Ortopedia e Traumatologia. Published by Thieme Revnter Publicações Ltda, Rio de Janeiro, Brazil 


\begin{abstract}
Keywords

- hallux valgus

- metatarsal bones

- radiography

- statistical analysis

Objective To determine if the sesamoids migrate laterally in the feet with hallux valgus or if they only appear to move, maintaining their relationship with the other forefoot structures.

Methods Anteroposterior radiographs of 80 patients (94 feet, all weight-bearing), from the period between 2015 and 2016, were evaluated. Forty-eight had a valgus hallux angle greater than $15^{\circ}$ (hallux valgus group) and 46 presented a hallux valgus angle lower than $15^{\circ}$ (control group). The distances from the first metatarsus head and the lateral sesamoid bone to the second metatarsus axis were measured. Subsequently, the coefficients of these distances were determined by the length of the second metatarsus to adjust it for different foot sizes.

Results Both the absolute and the relative measures from the first metatarsus head to the second metatarsus axis were significantly different between the groups, with a positive correlation with hallux valgus and intermetatarsal angles. However, neither the absolute nor the relative distance of the lateral sesamoid bone to the second metatarsus was different between the groups, as they did not correlate with hallux valgus or intermetatarsal angles.

Conclusion Despite the medial deviation of the first metatarsus in hallux valgus, the sesamoid bone maintains its relationship with the second metatarsus in the transverse plane. This apparent lateral displacement may lead to misinterpretation of these radiographs. This fact is of paramount importance in the pre-, intra-, and postoperative period of patients with hallux valgus.
\end{abstract}

\section{Introdução}

O hálux valgo (HV) é uma deformidade altamente prevalente nos pés, afeta $23 \%$ dos adultos e $35,7 \%$ da população idosa. ${ }^{1}$ É uma das queixas mais comuns nos pés dos pacientes que procuram os especialistas em cirurgia do pé e do tornozelo. Acomete predominantemente mulheres adultas. Suas causas são múltiplas, intrínsecas e extrínsecas. ${ }^{2,3}$ Além disso, as manifestações clínicas podem ser variadas com diferentes elementos anatômicos envolvidos. Embora seja uma desordem conhecida há séculos, ${ }^{4}$ ela é complexa e seu tratamento ainda não está totalmente padronizado, como demonstram centenas de técnicas cirúrgicas descritas na literatura, com resultados nem sempre uniformes. ${ }^{5,6}$

Os sesamoides do hálux são ossos acessórios do pé fundamentais para o funcionamento fisiológico da primeira articulação metatarsofalângica. Eles potencializam a força do músculo flexor curto do hálux, funcionam como "fulcro" e localizam-se sob a cabeça do primeiro metatarso, são ligados à base da primeira falange proximal pela placa plantar. ${ }^{4} \mathrm{O}$ deslocamento lateral dos sesamoides é frequentemente encontrado nos pés com hálux valgo. Tal desvio foi reconhecido desde o início deste século, no qual se acreditava que o realinhamento dos sesamoides para sua posição normal era um dos critérios para uma cirurgia bem-sucedida. ${ }^{7}$ Embora não fosse completamente explicado, já se observava que a migração lateral dos sesamoides em relação à cabeça do primeiro metatarso desempenhava um importante papel anatômico e funcional tanto no desenvolvimento inicial e progressão do transtorno quanto no potencial de recidiva pós-operatória. ${ }^{8}$
Apesar do longo decorrido no estudo da articulação metatarsofalângica na gênese e na progressão do hálux valgo, poucas investigações científicas que caracterizassem a mecânica dessa articulação e sua contribuição para o HV foram feitas. ${ }^{8}$ Em virtude disso, a avaliac,ão da posic,ão dos sesamoides na primeira articulação metatarsofalângica permanece em evidência visto sua importância para o diagnóstico, tratamento e recidiva do HV.

O objetivo do presente estudo foi determinar se os sesamoides migram lateralmente nos pés com $\mathrm{HV}$ ou se apenas aparentam deslocar-se e causam frequentemente uma interpretação errada das radiografias.

\section{Material e métodos}

\section{Pacientes}

Foi feito um estudo retrospectivo de pacientes atendidos em um único serviço de atendimento ortopédico. Todos os pacientes atendidos no setor de ortopedia devido a HV ou outras patologias nos pés de 2015 a 2016 foram elegíveis para participar do estudo. Pacientes menores de 18 anos, além daqueles que apresentavam deformidades nos pés, fise aberta, infecção, história de trauma ou cirurgia prévia foram excluídos.

Os pés foram radiografados na projeção anteroposterior (AP), de acordo com Rosemberg, ${ }^{9}$ ou seja, na posição ortostática, com o tubo angulado de $15^{\circ}$ em relação ao eixo vertical, o raio central sobre o osso navicular e a distância foco-filme de um metro. Pés com ângulo de hálux valgo (AHV) $>15$ o foram classificados com deformidade em hálux valgo; e aqueles com $\mathrm{AHV}<15$ o foram classificados como normais (controle). 


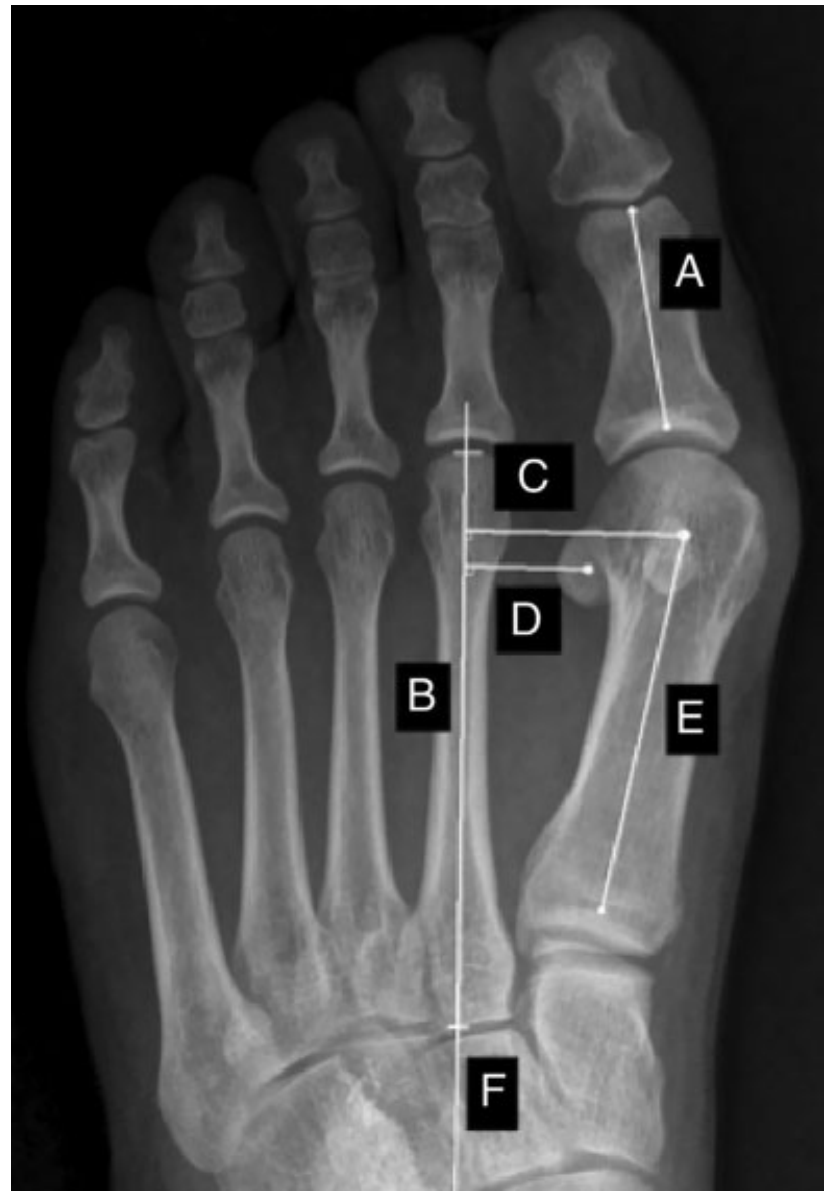

Fig. 1 Medições das relações dos sesamoides com os ossos do pé. Linha A - eixo da falange proximal; Linha B - comprimento do segundo metatarso; Linha C - distância entre a cabeça do primeiro metatarso e o eixo do segundo metatarso; Linha D - distância entre o centro do sesamoide lateral e o eixo do segundo metatarso; Linha $\mathrm{E}$ - eixo do primeiro metatarso; Linha $\mathrm{F}$ - eixo do segundo metatarso. $\mathrm{O}$ ângulo de hálux valgo é formado pelas linhas A e E, e o ângulo intermetatarsal é formado pelas linhas E e F.

\section{Medições}

As medições foram tomadas com o sistema digital PACS por dois dos autores (MD e GE). O AHV foi medido entre o eixo do primeiro metatarso e o eixo da falange proximal e o ângulo intermetatarsal (AIM) foi medido entre o eixo do primeiro metatarso e o eixo do segundo metatarso ( - Fig. 1 ). 0 eixo da falange proximal foi desenhado através do centro da super- fície articular proximal e o centro da extremidade distal da epífise. 0 eixo do segundo metatarso foi desenhado através do centro da superfície articular distal e do centro da extremidade proximal da diáfise. Finalmente, o primeiro metatarso foi desenhado para ligar o centro da cabeça ao centro da base do metatarso, como descrito por Miller. ${ }^{10} \mathrm{~A}$ posição do sesamoide lateral em relação ao segundo metatarso (PSM) foi definida como a menor distância do centro do sesamoide lateral até o eixo do segundo metatarso. A posição do primeiro metatarso em relação ao segundo metatarso (PMM) foi definida como a menor distância do centro da cabeça do primeiro metatarso até o eixo do segundo metatarso. Para adequar os diferentes tamanhos de pés estudados, foram definidos o coeficiente de desvio para o sesamoide lateral (CDSL) e o coeficiente de desvio para o metatarso (CDM).

$$
\mathrm{CDSL}=\mathrm{PSM} / \mathrm{CM} \text { e } \mathrm{CDM}=\mathrm{PMM} / \mathrm{CM}
$$

Onde CM é o comprimento longitudinal do segundo metatarso.

\section{Confiabilidade e análise estatística das medições}

Todos os pés do estudo foram medidos por dois autores (MD e GE) separadamente quanto ao AHV, AIM, PSM, PMM e CM. A correlação interobservador foi calculada a fim de se obter a confiabilidade das medições ( $\mathbf{- T a b e l a ~} \mathbf{1}$ ).

As medições de PMM, CDM, PSM, CDSL e CM foram feitas e comparadas entre os dois grupos (HV e controle) através dos testes $t$ de Student, Mann-Whitney e em alguns casos de Kolmogorov-Smirnov. As correlações de PMM, CDM, PSM, CDSL e CM com AHV e AIM foram feitas com os coeficientes e a matriz de correlação de Pearson. Todos os dados estavam de acordo com os pressupostos dos testes usados para analisálos (-Tabela 2). Valores de p menores do que 0,05 foram definidos como significantes. Os dados foram analisados com o pacote de software estatístico XLSTAT.

\section{Resultados}

\section{Informação demográfica}

Dos 80 pacientes envolvidos (94 pés), 48 pés (de nove homens e 39 mulheres) tinham HV. Sua média de idade foi de 49,25 (entre 18 e 86 ) anos. Os outros 46 pés (de 11 homens e 35 mulheres) não tinham HV e constituíram o grupo controle. Sua média de idade foi de 47,17 (entre 18 e 88)

Tabela 1 Confiabilidade das medições interobservadores na posição do sesamoide lateral no hálux algo

\begin{tabular}{|c|c|c|}
\hline Medições & $\begin{array}{l}\text { Correlação interobservador } \\
\text { (Hálux valgo) }\end{array}$ & $\begin{array}{l}\text { Correlação interobservador } \\
\text { (Pé normal) }\end{array}$ \\
\hline Ângulo de hálux valgo & 0,723 & 0,244 \\
\hline Ângulo intermetatarsal & 0,987 & 0,795 \\
\hline Posição do sesamoide lateral (PSM) ${ }^{\mathrm{a}}$ & 0,269 & 0,511 \\
\hline Posição da cabeça do 10 metatarso (PMM) ${ }^{\mathrm{b}}$ & 0,683 & 0,862 \\
\hline Comprimento do segundo metatarso & 0,472 & 0,381 \\
\hline
\end{tabular}

${ }^{a}$ Distância entre o centro do sesamoide lateral e o eixo do segundo metatarso.

bistância entre o centro da cabeça do primeiro metatarso e o eixo do segundo metatarso. 
Tabela 2 Medições nos pés com hálux valgo e nos pés normais (controle)

\begin{tabular}{|l|l|l|}
\hline Medições & $\begin{array}{l}\text { Grupo hálux } \\
\text { valgo }(\mathbf{n}=\mathbf{4 8})\end{array}$ & $\begin{array}{l}\text { Grupo controle } \\
\mathbf{( n = 4 6 )}\end{array}$ \\
\hline AHV (o) & $30,5(15$ a 54,65$)$ & $8,1(5,5$ a 15$)$ \\
\hline AIM (o) & $13,5(6,25$ a 18,3$)$ & $11(3,75$ a 14,85$)$ \\
\hline PSM $(\mathrm{mm})$ & $15,25(13,25$ a 24,2$)$ & $14,1(13,45$ a 20,45$)$ \\
\hline PMM (mm) & $25,4(20,8$ a 38,9$)$ & $20,5(18,3$ a 29,55$)$ \\
\hline CM $(\mathrm{mm})$ & $74,5(61$ a 89,5$)$ & $72,75(64,5$ a 88,15$)$ \\
\hline CDSL & $0,219(0,17$ a 0,273$)$ & $0,216(0,181$ a 0,295$)$ \\
\hline CDM & $0,375(0,299$ a 0,478$)$ & $0,331(0,269$ a 0,402$)$ \\
\hline
\end{tabular}

Abreviações: AHV, ângulo de hálux valgo; AIM, ângulo intermetatarsal; $\mathrm{CDM}$, coeficiente do primeiro metatarso (PMM/CM); CDSL, coeficiente de desvio do sesamóide lateral (PSM/CM); CM, comprimento do segundo metatarso; PMM, posição da cabeça do primeiro metatarso; PSM, posição do sesamóide lateral.

anos. Os grupos não tiveram diferença estatística em idade ou sexo ( $\mathrm{p}=0,891$ e $\mathrm{p}=0,245$, respectivamente).

\section{Medições e confiabilidade}

A média das diferenças entre os dois examinadores não foi diferente de zero pelo teste t de student nos dois grupos (HVe controle), o que levou à conclusão de que não houve diferença estatística nos resultados das medições dos examinadores $(\mathrm{p}>0,05)$. A distância absoluta entre o primeiro e o segundo metatarso (PMM) e o coeficiente calculado para equalizar a variação dos diferentes tamanhos de pés (CDM) mostraram diferença significativa entre os dois grupos (HV e controle). Ambos os valores foram maiores nos pés com HV, indicaram um deslocamento do primeiro metatarso em relação ao segundo. Além disso, tanto PMM quanto CDM se correlacionaram positivamente com AHV e AIM, ou seja, quanto maiores os valores de PMM ou CDM, maiores os valores de AHV e AIM (PMM-AHV, $\mathrm{r}=0,579$, $\mathrm{p}<0,0001$; PMM-AIM, $\mathrm{r}=0,630, \mathrm{p}<0,0001$; CDM-AHV, $\mathrm{r}=0,394$, $\mathrm{p}=0,006$; CDM-AIM, $\mathrm{r}=0,682, \mathrm{p}<0,0001)$.

Em adição, nem a distância do sesamoide lateral ao segundo metatarso (PSM) nem o coeficiente calculado como controle pela variação do tamanho dos pés (CDSL) mostraram diferença estatisticamente significante entre os dois grupos. Os valores medidos não aumentaram nos pés com $\mathrm{HV}$, indicaram que o sesamoide lateral permanece relativamente estável em relação ao segundo metatarso. Em adição, nem a medida de PSM nem a de CDSL se correlacionaram com AHV e AIM (PSM-AHV, $\mathrm{r}=0,184$, $\mathrm{p}=0,21 ; \quad$ PSM-AIM, $\mathrm{r}=0,173, \quad \mathrm{p}=0,24 ; \quad$ CDSL-AHV, $r=0,07, p=0,639 ;$ CDSL-AIM, $r=0,126, p=0,395)$.

\section{Discussão}

Durante o desenvolvimento do HV, há um desequilíbrio progressivo das estruturas que formam o antepé. Dessa forma, o tendão do abdutor do hálux é deslocado plantarmente e o manguito plantar intrínseco (constituído por adutor do hálux, flexores do hálux e abdutor do hálux), assim como o extensor longo do hálux, se desloca lateralmente e se torna uma força de adução no hálux. ${ }^{5,11}$ A rotação lateral contínua dessas estruturas leva ao deslocamento lateral ou rotação dos sesamoides em relação à superfície plantar do primeiro metatarso. ${ }^{12}$

Contudo, na realidade, acreditamos que o primeiro metatarso desvia medialmente com o desenvolvimento do HV enquanto os sesamoides permanecem presos ao segundo metatarso. Devido a essa relação entre os sesamoides e os metatarsos, diferentes métodos de avaliação radiográfica já foram usados na tentativa de estagiar e padronizar sua posição e seu deslocamento dentro dessa patologia. Tanto radiografias AP quanto radiografias tangenciais dos pés são descritas para tal. ${ }^{12,13}$ No entanto, o HV é uma deformidade abrangente, envolve todo o antepé. E tais estudos, até onde sabemos, não puderam encontrar a relação correta entre os sesamoides e o resto do pé, inclusive os raios laterais, no HV. ${ }^{14}$ A descrição dessa relação sistemática e matematicamente pode ajudar a caracterizar a deformidade do primeiro metatarso e dos demais raios do pé.

Dado que o segundo metatarso está mais próximo dos sesamoides e muitas vezes representa o eixo do antepé, ele foi escolhido como marco de referência em nosso estudo. Analisamos a posição do centro do sesamoide lateral e da cabeça do primeiro metatarso com e sem HV em relação a esse marco. Vários estudos já verificaram que as radiografias tangenciais com carga dos pés parecem ser melhores para avaliar a subluxação dos sesamoides, pois a rotação medial do metatarso no HV pode alterar o deslocamento percebido entre os sesamoides e o primeiro metatarso nas radiografias AP com carga dos pés. ${ }^{8,12,13}$ No entanto, nenhum desses estudos mostrou que há alteração da relação entre os sesamoides e os metatarsos laterais. Em contraste, a necessidade de dorsiflexão da articulação metatarsofalângica na visão tangencial pode alterar a posição dos sesamoides sob o primeiro metatarso. ${ }^{13}$ Portanto, escolhemos a visão radiográfica AP com carga dos pés para evitar esse problema e para tornar mais conveniente a medição do AHV e do AIM.

Embora a subluxação sobre os sesamoides eventualmente possa ocorrer no HV, a distância entre o sesamoide lateral e o segundo metatarso (PSM) não foi significativamente maior no grupo HV do que no grupo controle no nosso estudo. Além disso, essa distância não se correlacionou nem com AHV nem com AIM. Todavia, no grupo HV, a distância entre o primeiro e o segundo metatarsos (PMM) foi expressivamente maior do que no grupo controle. E também houve uma correlação positiva dessa distância com AHV e AIM. Ademais, as distâncias relativas dessas medidas (CDM e CDSL) também mostraram a mesma relac,ão que as distâncias absolutas (PSM e PMM) entre os dois grupos. Acreditamos também que tais medições (CSDL e CDM) possivelmente expressem mais objetivamente essas relações e correlações, visto que levam em consideração as medições com os diferentes tamanhos de pés dos pacientes estudados.

Nossos dados mostraram que os sesamoides mantêm sua relação com o segundo metatarso, mesmo nos pés com HV. Em contrapartida, o deslocamento medial do primeiro metatarso cria a chamada "subluxação do sesamoide". Embora 
esse fenômeno tenha sido amplamente pesquisado, poucos eram baseados em cima de estudos prospectivos, especialmente a partir da análise da relação entre o centro do sesamoide lateral e os metatarsos laterais nos pés com HV e normais (controle). Geng et al., ${ }^{14}$ assim como em nosso estudo, demonstraram que o sesamoide lateral mantém sua relação com o segundo metatarso no plano transverso. Seu estudo também foi feito através de radiografias AP com carga dos pés, tanto com HV quanto normais (controle). Além disso, corroboraram nossos achados de que, com a padronização das medições por coeficientes, através dos diferentes tamanhos dos pés, se comprovou que o sesamoide mantém sua posição em relação ao segundo metatarso, enquanto que a posição da cabeça do primeiro metatarso desloca-se medialmente nos pés com HV.

Saragas e Becker ${ }^{15}$ não encontraram diferença significativa na distância entre a borda lateral do sesamoide lateral e o segundo metatarso em pés com e sem HV. Entretanto, eles não avaliaram sua correlação com ângulos tradicionalmente medidos nos pés, como AHV e AIM. Além disso, também não calcularam a distância relativa com o ajuste de acordo com os diferentes tamanhos de pés estudados. Por fim, fizeram as medições a partir da borda lateral do sesamoide. Contudo, como existem protrusões irregulares na borda lateral do sesamoide, acreditamos que essa medição nem sempre consegue obter a localização com precisão e acurácia necessária ao estudo.

Huang et al. ${ }^{16}$ demonstraram que a localização dos sesamoides nos pés com HV não se altera em relação ao segundo metatarso após a correção cirúrgica. No entanto, eles também escolheram a borda lateral do sesamoide lateral como marco para a medição e não compararam a localização do sesamoide entre os pés com e sem HV. Além disso, concordamos com Geng et al. ${ }^{14}$ que a comparação da localizac,ão pré e pós-operatória dos sesamoides pode ser influenciada pelo próprio procedimento cirúrgico, ou mesmo pelo cirurgião que faz o procedimento.

Ramdass e Meyr ${ }^{17}$ e Judge et al. ${ }^{18}$ escolheram o sesamoide medial e o segundo metatarso como pontos de referência nos pés com HV e relataram que a distância não mudou nos planos transverso e frontal pós-operatório. No entanto, essa distância é maior do que entre o sesamoide lateral e o segundo metatarso. Assim, acreditamos que o primeiro é uma medida menos sensível da localização do sesamoide e poderia ocultar pequenas diferenças.

Katsui et al. ${ }^{19}$ também estudaram o alinhamento do sesamoide medial e a relação entre seu mau alinhamento e as alterações degenerativas na articulação metatarsossesamoide. Entretanto, além de imagens de radiografia AP dos pés, também usaram imagens de tomografia computadorizada dos pés de pacientes com HV. Não avaliaram, porém, se essas alterações também estavam presentes nos pés sem HV, pois não houve um grupo controle. Ao contrário dos achados de nosso estudo, eles observaram que houve um deslocamento lateral progressivo do sesamoide de acordo com o aumento da deformidade do HV. Da mesma maneira, verificaram que esse deslocamento lateral do sesamoide estava associado ao agravamento das alterações degenerativas da articulação metatarsossesamoide.
Ao corrigir o HV, o primeiro metatarso deve ser trazido de volta para cima dos sesamoides, e não vice-versa. Normalmente são necessários procedimentos adicionais de liberação de tecidos moles. Contudo, até hoje, muitos cirurgiões fazem essa liberação em diferentes tecidos através de técnicas cirúrgicas diferentes. ${ }^{20}$ Não há um padrão ou regra.

\section{Conclusão}

Em conclusão, não encontramos alteração significativa na posição do sesamoide lateral em relação ao segundo metatarso nos pés com HV e normais (controle). Esse aparente deslocamento lateral do sesamoide nos pés com HV é uma interpretação errada das radiografias na incidência AP dos pés dos pacientes. Dessa forma, os sesamoides mantêm sua relação com o segundo metatarso e o desvio medial do primeiro metatarso, e não a migração lateral dos sesamoides, é o que leva à subluxação entre o primeiro metatarso e os sesamoides. Portanto, a "subluxação metatarsiana” poderia descrever melhor tanto a subluxação da articulação metatarsossesamoide quanto o desalinhamento da primeira articulação metatarsofalângica.

Conflitos de interesse

Os autores declaram não haver conflitos de interesse.

\section{Referências}

1 Nix S, Smith M, Vicenzino B. Prevalence of hallux valgus in the general population: a systematic review and meta-analysis. J Foot Ankle Res 2010;3(01):21

2 Pansini JV, Matunaga RY, Aguiar A, Buchen CE. Hálux valgo: tratamento com osteotomias tipo Chevron. Rev ABTPe 2008;2 (01):17-22

3 Nix SE, Vicenzino BT, Collins NJ, Smith MD. Characteristics of foot structure and footwear associated with hallux valgus: a systematic review. Osteoarthritis Cartilage 2012;20(10):1059-1074

4 CoCoughlin MJ. Sesamoids and accessory bones of the foot. In: Mann RA, Coughlin MJ, editors. Surgery of the foot and ankle. 9 th edition. St Louis (MO): Mosby; 2007:531-610

5 Coughlin MJ. Hallux valgus. J Bone Joint Surg Am 1996;78(06): 932-966

6 Nery CAS. Hálux valgo. Rev Bras Ortop 2001;36(06):183-200

7 Silver D. The operative treatment of hallux valgus. J. Bone Joint Surg. 1923;5:225-232

8 Talbot KD, Saltzman CL. Assessing sesamoid subluxation: how good is the AP radiograph? Foot Ankle Int 1998;19(08): $547-554$

9 Rosemberg LA. Estudo radiológico do pé. In: Salomão O, Carvalho Junior AE, editors. Pé e tornozelo [apostila]. São Paulo: Instituto de Ortopedia e Traumatologia - HC/FMUSP; 1994:19-27

10 Miller JW. Distal first metatarsal displacement osteotomy. Its place in the schema of bunion surgery. J Bone Joint Surg Am 1974;56(05):923-931

11 Coughlin MJ, Jones CP. Hallux valgus: demographics, etiology, and radiographic assessment. Foot Ankle Int 2007;28(07):759-777

12 Kuwano T, Nagamine R, Sakaki K, Urabe K, Iwamoto Y. New radiographic analysis of sesamoid rotation in hallux valgus: comparison with conventional evaluation methods. Foot Ankle Int 2002;23(09):811-817

13 Yildirim Y, Cabukoglu C, Erol B, Esemenli T. Effect of metatarsophalangeal joint position on the reliability of the tangential 
sesamoid view in determining sesamoid position. Foot Ankle Int 2005;26(03):247-250

14 Geng X, Zhang C, Ma X, Wang X, Huang J, Xu J, et al. Lateral sesamoid position relative to the second metatarsal in feet with and without hallux valgus: a prospective study. J Foot Ankle Surg 2016;55(01):136-139

15 Saragas NP, Becker PJ. Comparative radiographic analysis of parameters in feet with and without hallux valgus. Foot Ankle Int 1995;16(03):139-143

16 Huang EH, Charlton TP, Ajayi S, Thordarson DB. Effect of various hallux valgus reconstruction on sesamoid location: a radiographic study. Foot Ankle Int 2013;34(01):99-103

17 Ramdass R, Meyr AJ. The multiplanar effect of first metatarsal osteotomy on sesamoid position. J Foot Ankle Surg 2010;49(01): 63-67
18 Judge MS, LaPointe S, Yu GV, Shook JE, Taylor RP. The effect of hallux abducto valgus surgery on the sesamoid apparatus position. J Am Podiatr Med Assoc 1999;89(11-12):551-559

19 Katsui R, Samoto N, Taniguchi A, Akahane M, Isomoto S, Sugimoto $\mathrm{K}$, et al. Relationship Between Displacement and Degenerative Changes of the Sesamoids in Hallux Valgus. Foot Ankle Int 2016; 37(12):1303-1309

20 Fakoor M, Sarafan N, Mohammadhoseini P, Khorami M, Arti H, Mosavi S, et al. Comparison of clinical outcomes of scarf and chevron osteotomies and the mcbride procedure in the treatment of hallux valgus deformity. Arch Bone Jt Surg 2014;2(01): 31-36

21 Lin I, Bonar SK, Anderson RB, Davis WH. Distal soft tissue release using direct and indirect approaches: an anatomic study. Foot Ankle Int 1996;17(08):458-463 